\title{
Nocuous Skin Manifestations of Spironolactone: Drug Induced Leukocytoclastic Vasculitis
}

\author{
Awad Magbri*, Saad Rashid, Zach Crowe and Shaukat Rashid
}

Toledo Vascular Access Center, Toledo OH, USA

Received: April 10, 2018; Accepted: May 07, 2018; Published: May 11, 2018

*Corresponding author: Awad Magbri, Toledo Vascular Access Center, Toledo OH, USA, Tel: 815-520-8211; E-mail: elmagbri@hotmail.com

\begin{abstract}
The author discusses a case of drug induced vasculitis caused by spironolactone which is very rare complication of the drug. The authors give account on the type of vasculitis caused by drug induced allergy and also the differential diagnoses if the presentation is unusual with only hematuria and kidney injury. The benefits of Spironolactone and the side effects are also illustrated.
\end{abstract}

Key words: Vasculitis; Drug rash; Spironolactone; Hyperkalemia; ANCA associated Vasculitis; Cryoglobulinemia; Leukocytoclastic Vasculitis; Hypocomplementemia;

\section{Case History}

83 year-old Caucasian female with past medical history of hypertension, chronic kidney disease stage 3-4 secondary to hypertensive nephrosclerosis with minimal proteinuria, hypokalemia, and Sjugrene syndrome without extra-glandular involvement. The patient was admitted to hospital because of urinary tract infection and edema of the lower extremities. She was treated with antibiotics, and diuretics for the edema. Her blood pressure was not optimum at this visit and spironolactone $25 \mathrm{mg}$ was added to her medication to control her BP, hypokalemia, and proteinuria. She was on amlodipine, frusemide, clonidine, Irbesartan, and potassium supplement. The treating nephrologist decided to try spironolactone for better control of high BP and hypokalemia and phased out the potassium supplementation. At this encounter her micro-albumin-creatinine ratio was $379.3 \mathrm{mg} /$ gr, (normal value $<29.9 \mathrm{mg} /$ gr creatinine). Physical examination was otherwise normal except bilateral leg edema, and high blood pressure $(149 / 65 \mathrm{mmHg})$. Her estimated glomerular filtration rate (eGFR) was $28 \mathrm{ml} / \mathrm{min}$ ).

She returned to the nephrology clinic after 2 weeks for bilateral lower extremities itch, burning and painful rash involving the buttocks, upper thighs and shins bilaterally as shown in the figure 1-2. The rash was palpable, purpuric nodules symmetrically distributed over the lower extremities. She was sending for dermatological opinion and skin biopsy of the rash. The biopsy was consistent with leukocytoclastic vasculitis. On further questioning the patient, she recalled that she had had a similar rash long time ago when she was placed on "aldactone" and the doctor had to stop the medication because of the rash, subsequently, the rash went away. This triggered discontinuation of the spironolactone and she was treated with prednisone and topical steroids by the dermatologist. The current rash faded away in 3-4 weeks; however, she was still on $5 \mathrm{mg}$ of prednisone when she was last seen in the clinic. Her laboratory values are illustrated in table-1.

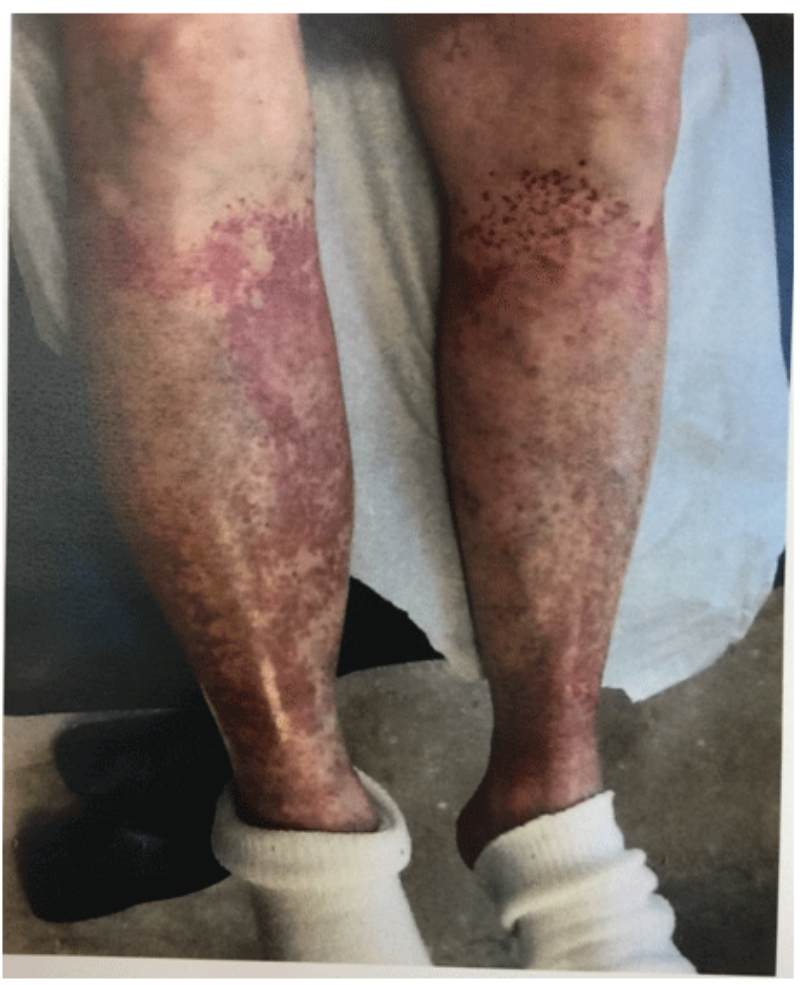

Figure 1: Leukocytoclastic vasculitis on the lower extremities

\section{Case Discussion}

Spironolactone is potassium sparing mineral corticoid receptor antagonist (MRA) which acts on the distal tubules and collecting ducts of the kidneys and antagonizing the effect of aldosterone, thereby causing inhibition of sodium and chloride reabsorption, and potassium secretion in the distal tubules. The bioavailability of spironolactone is $73 \%$, and it is $>90 \%$ proteinbound. The drug is extensively metabolized in the liver and 


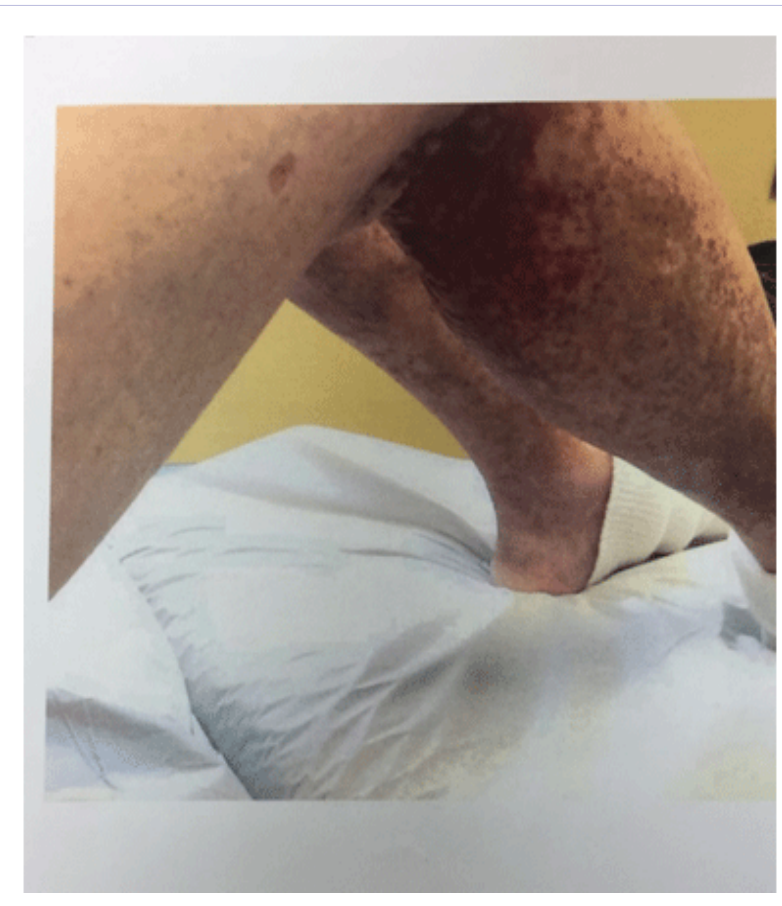

Figure 2: Leukocytoclastic vasculitis on the lower extremities excreted by renal (47-57\%), bile and eventually fecal route (35$41 \%)$. The elimination half-life of the drug ranges from 1.4 to 15 hours depending on the type of metabolites. Itis indicated for heart failure with reduced ejection fraction, hypertension especially when associated with hyperaldosteronism, hypokalemia, precocious puberty, hirsutism and female virilization syndrome. The American geriatric association (AGS) recommends that the drug should be avoided in patients $>65$ years old when creatinine clearance $<30 \mathrm{ml} /$ minute due to increased potassium levels associated with the medication [1]. Spironolactone has also been used for liver cirrhosis with ascites and diabetic nephropathy with proteinuria. It can be added to patients with metabolic alkalosis with hypokalemia due to diuretic use.

In renal impairment the drug can be used only if the potassium is low and the (eGFR is $>30 \mathrm{~mL} /$ minute). The drug is very effective for hypertension associated with hyperaldosteronism. There is black box warning that spironolactone has been shown to be tumorigenic in chronic toxicity studies in rats. It is contraindicated in acute renal insufficiency with low GFR $(<30 \mathrm{~mL} /$ minute $)$, Addison disease, hyperkalemia, or other conditions associated with high potassium.

Painful gynecomastia, hyperkalemia, hypomagnesemia, hyponatremia, or hypocalcemia are rare side effects of the

Table 1: Laboratory data of the patient during the 4 visitis

\begin{tabular}{|c|c|c|c|c|}
\hline Test & $1^{\text {st }}$ visit & $2^{\text {nd }}$ visit & $3^{\text {rd }}$ visit & $4^{\text {th }}$ visit \\
\hline WBC (4.2-9.5) & 7.6 & 9.0 & 9.1 & 8.9 \\
\hline Hemoglobin $(12-15 \mathrm{gr} / \mathrm{dl})$ & 11.7 & 11.5 & 11.9 & 11.9 \\
\hline Hematocrit (35.3-43.6\%) & 34.8 & 34.7 & 35.8 & 35.8 \\
\hline Glucose (74-106 gr/dl) & 96 & 75 & 74 & 108 \\
\hline BUN (7-18 mg/dl) & 27 & 18 & 32 & 35 \\
\hline Creatinine $(0.55-1.02 \mathrm{mg} / \mathrm{dl})$ & 1.35 & 1.52 & 1.82 & 1.50 \\
\hline Calcium $(9-10 \mathrm{mg} / \mathrm{dl})$ & 9.2 & 9.6 & 9.4 & 9.5 \\
\hline Sodium (136-145 mEq/dl) & 136 & 136 & 134 & 136 \\
\hline Potassium (3.5-5.1 mEq/dl) & 3.8 & 4.1 & 3.6 & 4.2 \\
\hline Chloride (98-107 mEq/dl) & 96 & 97 & 96 & 98 \\
\hline Carbone dioxide (21-32 mEq/dl) & 34 & 32 & 31 & 32 \\
\hline Anion gap (3-11 mEq/dl) & 6 & 7 & 7 & \\
\hline PTHI (11-67 pg/ml) & 79.7 & & 32.1 & 55.8 \\
\hline ANCA & & Negative & & \\
\hline ANA & & Negative & & \\
\hline Complement & & normal & & \\
\hline Cryoglobulins & & negative & & \\
\hline
\end{tabular}


drug. It is also contra-indicated in pregnancy but compatible with breastfeeding and lactation. Spironolactone has been used in heart failure with reduced ejection fraction $(<35 \%)$, and after myocardial infarction with either symptomatic heart failure or diabetes mellitus and left ventricular ejection fraction (LVEF<40\%).

The adverse effects of spironolactone are estimated to be $1-10 \%$ and include the followings

- Gynecomastia (9\%)

- Frequency not defined;

- Vasculitis

- Ataxia, confusion, dizziness

- Alopecia, erythematous maculopapular rash, pruritus, StevenJohnson syndrome, toxic epidermal necrolysis, urticarial

- Electrolyte disturbances

- Erectile dysfunction, impotence, mastalgia

- Agranulocytosis, leukopenia, thrombocytopenia

- Hepatic toxicity

- DRESS syndrome

- Increased blood urea nitrogen, renal failure and fever

Leukocytoclastic vasculitis is an uncommon side effect of spironolactone. It is a histological term that describes small vessel involvement of the subcutaneous tissue with inflammatory infiltrate which is composed of neutrophils [2-6].

It is mainly caused by drugs like phenytoin, allopurinol, nonsteroidal anti-inflammatory medications and many antibiotics [7-10], infections with hepatitis C and cryoglobulinemia and malignancy can also be offenders.

Many times the vasculitis appear after 9-12 days after exposure to the medication. Arthralgia, arthritis, and fever occurring at the same time as the typical rash have been described. Cutaneous small vessel vasculitis (CSVV) with petechial, purpuric eruptions which is non-blanching involving mainly the lower extremities and buttocks are classic presentation of the drug $[11,12]$. Evidence for circulating immune complex formation including hypocomplementemia, and deposition of the immune complex in the vessel wall of the subcutaneous tissues can be detected by immunofluorescence (IF) [13-15].This type of vasculitis is attributed to type-III immunological reaction. A lapsing period of 10-14 days is needed for the antigen-antibody deposition in the skin with appearance of the vasculitic rash. Involvement of the visceral and vital organs can sometimes accompany the cutaneous rash.

Low serum complement levels, especially low C4, may be present in mixed cryoglobulinemia and systemic lupus erythematosus vasculitis. The presence of anti-neutrophil cytoplasmic antibodies (ANCA) is indicative of ANCA associated vasculitis. These diseases have to be ruled out when evaluating a vasculitic rash, and pertinent laboratory tests have to be ordered.
The diagnosis of CSSV is usually suggested by the clinical findings and by the history of drug exposure or infection [12]. Immunofluorescence of the skin biopsy can sometimes demonstrate the deposition of immunoglobulin G (IgG), IgM and/or complement. The absence of immune deposition in the histological specimen, however, does not exclude immune complex vasculitis and can be attributed to late timing of biopsy relative to the skin rash, or may be associated with cases of pauciimmune type of vasculitis such as microscopic polyangiitis. If IgA is deposited in the skin on IF microscopy together with gastrointestinal and joint involvement, IgA vasculitis and HenochSchonlein purpura (HSP) should be considered. Non-specific findings like high erythrocyte sedimentation rate (ESR), C reactive protein, and anemia are common findings with the drug rash.

The differential diagnosis should include systemic vasculitis (ANCA-associated), malignancy which is frequently due to lymph proliferative disease, and infection.

Follow up of the case revealed that the rash faded away in 3-4 weeks; however, she was still on $5 \mathrm{mg}$ of prednisone when she was last seen in the clinic. Her kidney function has reverted to baseline after the drug was withdrawn and her blood pressure is well controlled on her current medications.

Conclusion: This case showed a rare side-effect of spironolactone in the form of vasculitis without visceral involvement. The treatment is focused on excluding systemic involvement and symptomatic management of the rash. Prednisone can sometimes be used to haste the healing process. Removing the offending drug is of paramount importance.

\section{References}

1. Fick DM, Semla TP, Beizer J, Brandt N, Dombrowski R, DuBeau CE, et al. The American Geriatrics Society 2015. Updated Beers Criteria for Potentially Inappropriate Medication Use in Older Adults. J Am Geriatric Soc. 2015;63(11):2227-2246. doi: 10.1111/jgs.13702

2. Jennette JC, Falk RJ, Andrassy K, Bacon PA, Churg J, Gross WL, et al Nomenclature of systemic Vasculitides. Proposal of an international consensus conference. Arthritis Rheum. 1994;37(2):187-192.

3. Carlson JA. The histological assement of cutaneous vasculitis. Histopathology. 2010;56(1):3-23. doi: 10.1111/j.13652559.2009.03443.x

4. Gonzalez-Gay MA, Garcia-Porrua C, Pujol RM. Clinical approach to cutenous vasculitis. Curr Opin Rheumol. 2005;17(1):56-61.

5. Jennette JC, Falk RJ. The role of pathology in the diagnosis of systemic vasculitis. Clin Exp Rheumatol. 2007;25(1 Suppl 44):S52-56.

6. Nagai Y, Hasegawa M, Igarashi N, Tanaka S, Yamanaka M, Ishikawa O. Cutaneous manifestations and histological features of microscopic polyangiitis. Eur J Dermatol. 2009;19(1):57-60. doi: 10.1684/ ejd.2008.0566

7. Mullick FG, McAllister HA Jr, Wagner BM, Fenoglio JJ Jr. Drug related vasculitis. Clinicopathologic correlations in 30 patients. Hum Pathol. 1979;10(3):313-325.

8. Martinez-Taboada VM, Blanco R, Garcia-Fluentes M, RodriguezValverde V. Clinical features and outcome of 95 patients with hypersensitivity vasculitis. Am J Med. 1997;102(2):186-191. 
9. Sais G, Vidaller A, Jucgla A, Gallardo F, Peyríf. Colchicine in the treatment of cutaneous leukocytoclastic vasculitis, Results of a prospective, randomized controlled trial. Arch Dermatol. 1995;131(12):13991402.

10. Blanco R, Martinez-Taboada VM, Rodriguez-Valverde V, GarciaFuentes M. Cutanepus vasculitis in children and adults. Asscociated diseases and etiologic factors in 303 patients. Medicine (Baltimore). 1998;77(6):403-416.

11. Parker CW. Allergic reactions in man. Pharmacol Rev. 1982;34(1):85104.

12. Loricera J, Blanco R, Ortiz-Sanjuan F, Hernández JL, Pina T, GonzálezVela MC, et al. Single-organ cutaneous small-vessel vasculitis according to the 2012 revised International Chapel Hill Consensus
Conference Nomenclature of Vasculitides: a study of 60 patients from a series of 766 cutaneous vasculitis cases. Rheumatology (Oxford). 2015;54(1):77-82. doi: 10.1093/rheumatology/keu295

13. Tosca N, Stratigos JD. Possible pathogenetic mechanisms in allergic cutaneous vasculitis. Int J Dermatol. 1988;27(5):291-296.

14. Van Rossum AP, Pas HH, Fazzini F, Huitema MG, Limburg PC, Jonkman MF, et al. Abundance of the long pentraxin PTX3 at sites of leukocytoclastic lesions in patients with small-vessel vasculitis. Arthritis Rheum. 2006;54(3):986-991.

15. Leavitt RY, Fauci AS. Polyangiitis overlap syndrome. Classification and prospective clinical experience. AM J Med. 1986;81(1):79-85. 